\title{
Modeling Gate Oxide Short Defects in CMOS Minimum Transistors
}

\author{
M. Renovell, J.M. Gallière, F. Azaïs and Y. Bertrand \\ Laboratoire d'Informatique Robotique Microélectronique de Montpellier LIRMM-UMII \\ Université de Montpellier II: Sciences et Techniques du Languedoc \\ UMR C5506 CNRS - 161, rue Ada 34392 Montpellier Cedex 5 France \\ Tel: (33)467418523 - Fax: (33)467418500 - Email: renovell@lirmm.fr
}

\begin{abstract}
In this paper a new model is proposed for Gate Oxide Short defects based on a non-split MOS transistor. Because the MOS is not split, this model allows to simulate minimum transistors in realistic digital circuits. The construction of the model is presented in details using a comprehensive and didactic approach. It is demonstrated that the electrical behavior of the proposed model matches in a satisfactory way the defective transistor behavior.
\end{abstract}

\section{Introduction}

$\boldsymbol{T}_{\text {he advent of integrated circuit technology has }}$ introduced electronics in many aspect of present-day life. As the use of electronic components increases, the expectation of lower cost, better accuracy, and higher reliability increases. Lower cost and better accuracy is achieved by putting more transistors per unit of silicon, using design automation, increasing device operation speed, and reducing its power consumption. However, these design steps cannot guarantee reliability. In fact, as the circuit density increases, the probability of a manufacturing defect increases. The higher expectation of reliability can only be met by more thorough and comprehensive testing.

Due to the complexity of IC technological process, many physical defects occur during the manufacturing of any system. The typical defects encountered in today technologies and modeled in yield simulators are the socalled spot defects that may cause shorts and/or breaks at one or more of the different conductive levels of the devices. Test generation for any type of defect is obviously not feasible due to the huge amount of CPU time and memory size required. Instead, test generation relies on fault models that are supposed to both represent the defect behavior and allow easy generation of test vectors.

Classical fault models (stuck-at, stuck-open, stuckon, ...) have been proved to be efficient for the analysis of many of these faults. However, it is well-known that these fault models cover only partially the spectrum of real failures in today's integrated circuits. The increasing demand of low ppm defect rates requires the derivation of ever more accurate fault models. In particular, a special attention must be paid to defects that exhibit complex behavior not accurately represented by classical fault models and defects with a high probability of occurrence. Gate Oxide Short (GOS) defects belong to both categories since (i) they change some of the electrical features of the transistor, and (ii) they are predominant defects in today technologies in which devices are scaled down and oxide thickness reduced. A number of research works have been conducted in the past years dealing with the electrical characterization [1-10] and modeling [11-17] of this kind of failure.

Both for the characterization and modeling process, one should distinguish between two different types of gate oxide short, i.e. GOS connecting directly the gate to the drain or source or GOS connecting the gate to the channel. For the first type of defect, a realistic model based on the addition of a short resistance between the gate the drain (or source) has been proposed in [12]. For the second type of defect, several models have been proposed based on the split of the faulty device in several smaller devices [1114]. Although these models perfectly match the electrical characteristics of the defect behavior, they suffer from a strong limitation: they cannot be used to study GOS defects in realistic digital CMOS circuits. Indeed, these models are not able to cope with minimum transistors affected by a GOS failure. However, most of the transistors involved in a digital cell library are today designed at minimum-size. It is therefore the objective of this paper to present a new model permitting to handle minimum transistors affected by gate-to-channel shorts.

The paper is organized as follows. GOS failures and their associated behavior are described in section 2 . Defect modeling is addressed in section 3 and limitations of the previously proposed models are discussed. In section 4, we introduce the new non-linear non-split model and show how this model permits to accurately represent the defect behavior while preserving the length of the original transistor. Finally, concluding remarks are given in section 5 . 


\section{GOS Failures}

\subsection{Defect description}

A GOS is a transistor defect that cause a relatively low impedance path between the CMOS gate and the underlying silicon. Depending on the defect location, the GOS can be seen as a short circuit between the gate and transistor channel or as a short circuit between the gate and drain or source diffusion zones. Figure 1 illustrates these two types of defect. For both types, an undesired path of current through the oxide of the gate appears thus creating a violation of the gate isolation principle. It is generally admitted that GOS may have different origins such as lithographic defect on mask, field failure due to ESD...

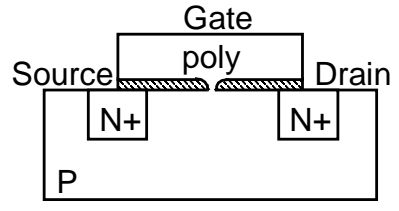

(a) gate-to-channel

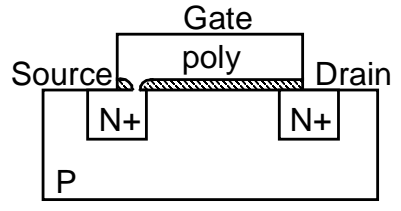

(b) gate-to-source (drain)
Fig.1: Pinhole in a MOS gate

\subsection{Defect behavior}

$\boldsymbol{O}$ ne of the main specificity of a GOS defect is that the pinhole that shorts the gate to one point of the underlying silicon creates a new device in which an important gate current can flow. It is therefore possible to study the IG vs VG characteristics of a defective transistor. As commented by the authors of many previous papers [1-3], two distinct electrical behaviors are observed depending on the defect type. The first type of defects connecting the gate to drain or source diffusion zone is referred as an ohmic defect and exhibits a linear behavior in the IG vs VGS characteristics. In contrast, the second type of defects connecting the gate to transistor channel exhibits a non-linear behavior in the IG vs VGS characteristics. Figure 2 shows typical IG vs VGS characteristics for both the ohmic and non-ohmic types of defects.

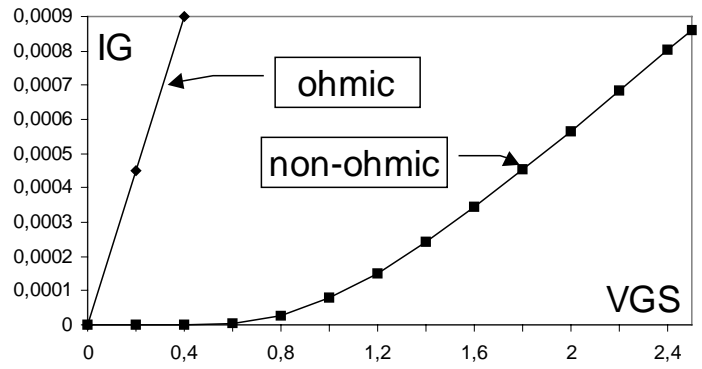

Fig.2: IG vs VGS characteristics of a MOS with a GOS
Regarding the ID vs VDS characteristics, all experiments have demonstrated that the drain current of a defective transistor only slightly resemble the typical MOS transistor drain current. Figure 3.a and 3.b show the typical ID vs VDS characteristics of non-defective and defective transistors. Basically, the defect manifests itself through two main phenomena:

- reduction of the maximum drain current at high VDS,

- appearance of a negative drain current when VDS is small in comparison with VGS.

For a given VGS, we will denote IDmax the maximum drain current and IDmin the minimum drain current at VDS $=0$.

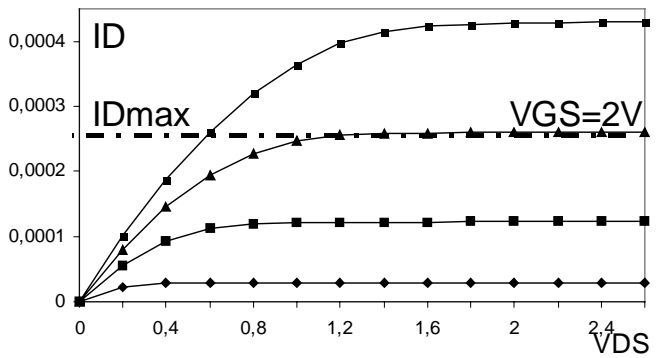

(a) non-defective NMOS

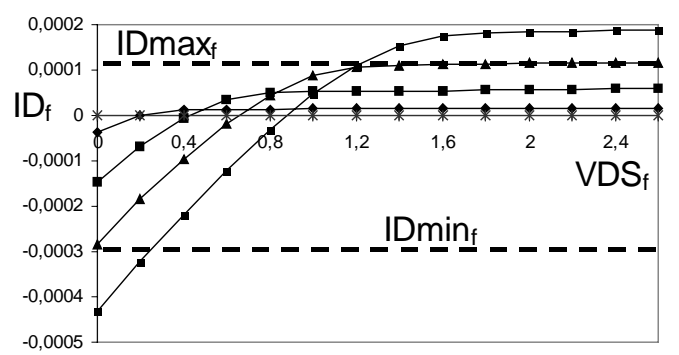

(b) NMOS with a gate-to-channel GOS Fig.3: ID vs VDS characteristics

\section{Electrical MOS models of GOS}

\subsection{Linear Non-Split MOS Model}

$\boldsymbol{A}$ simple linear model has been proposed in [12] based on the addition of a short resistance between the gate and drain or source electrodes as illustrated in figure 4 . This model is perfectly adequate to represent GOS defects connecting the gate to drain or source diffusion zone in a n-channel transistor. This model has been extended in [15-17] to cope with both $n$ - and $p$ channel transistors.
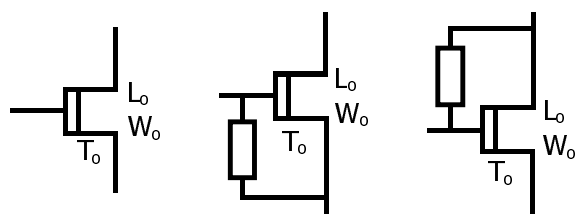

original transistor gate-to-source short gate-to-drain short

Fig.4: Linear non-split MOS model 
One main advantage of these models is that the length of the original transistor $T_{0}$ is preserved, implying that the model can handle minimum transistors. However, these models cannot apply for gate oxide shorts between the gate and the channel of a transistor as they present a different set of electrical properties. In particular, they are not able to represent the non-linear behavior depicted in the IG vs. VGS characteristics of figure 2.

\subsection{Non- Linear Split MOS Model}

\section{$I_{n}$ order to study GOS defects connecting the gate to}

the channel, an electrical model of the fault-free transistor based on a lumped-element model has been proposed in [11]. In this model represented in figure 5, the nondefective channel is split and become a two-dimensional array of MOS transistors.

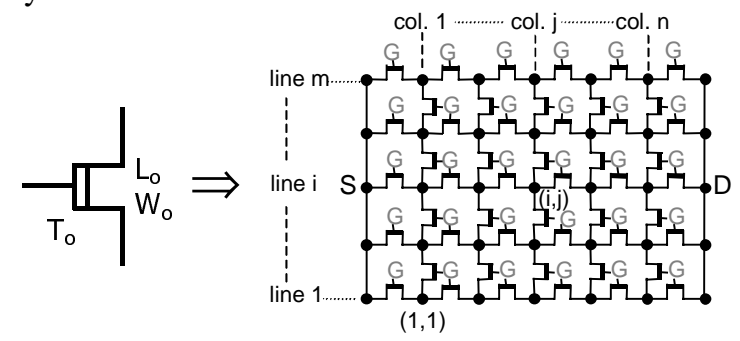
(a) original MOS
(b) $5 \times 5$ split model

Fig.5: Split model of a non-defective NMOS

As an example, we consider an original transistor $\mathrm{T}_{\mathrm{o}}$ with $\mathrm{L}_{\mathrm{o}}=2.1 \mu \mathrm{m}$ and $\mathrm{W}_{\mathrm{o}}=3.5 \mu \mathrm{m}$ in a $0.25 \mu \mathrm{m}$ technological process. This transistor can be split in a $5 \times 5$ network of elementary NMOS transistors designed at minimal length $\mathrm{L}_{\mathrm{i}}=0.25 \mu \mathrm{m}$ and $\mathrm{W}_{\mathrm{i}}=0.5 \mu \mathrm{m}$. As illustrated in figure 6 , a good agreement is observed between the simulated ID vs VDS characteristics of the $5 \times 5$ network and the original transistor.

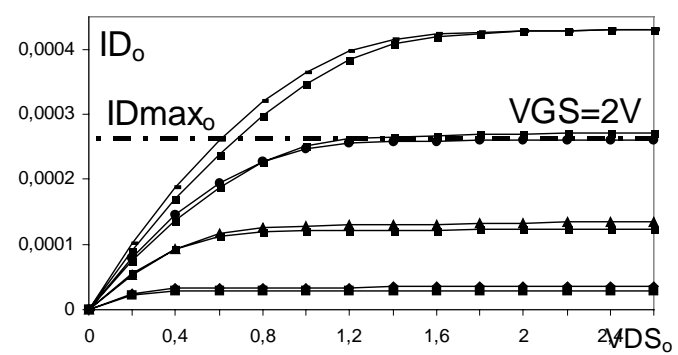

Fig.6: Comparison of the ID vs VDS characteristics of the original transistor and the split MOS model

Based on this lumped-element model, a GOS defect can be introduced in the transistor by connecting a short resistance $R_{G O S}$ between the common gate $G$ and one of the internal nodes of the network, denoted $\left(\mathrm{i}_{\mathrm{f}}, \mathrm{j}_{\mathrm{f}}\right)$. Figure 7 gives an example of a central defect introduced in the transistor. The defect resistance and location can then be varied through the value of $\mathrm{R}_{\mathrm{GOS}}$ and position $\left(\mathrm{i}_{\mathrm{f}}, \mathrm{j}_{\mathrm{f}}\right)$ in the network.

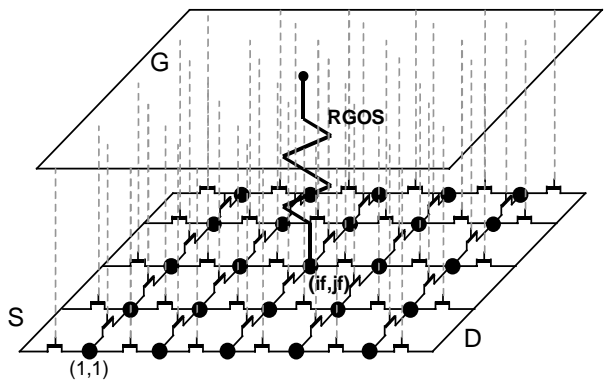

Fig.7: Split model of a NMOS with a GOS

This model has been demonstrated to perfectly describe the electrical behavior of gate-to-channel shorts [8-9]. For illustration, figure 8.a shows the simulated ID vs VDS characteristics of a defective transistor with a $1 \Omega$ central defect. For a given VGS, for instance VGS=2V, we observe a reduction of the maximum drain current IDmax $_{\mathrm{f}}$ compared to the non-defective one IDmax together with the appearance of a negative drain current for small VDS. This model also permits to simulate the IG vs VGS characteristics, as depicted in figure 8.b. Note that these characteristics are in agreement with the experimental behavior observed for gate-to-channel shorts (see figure 2).

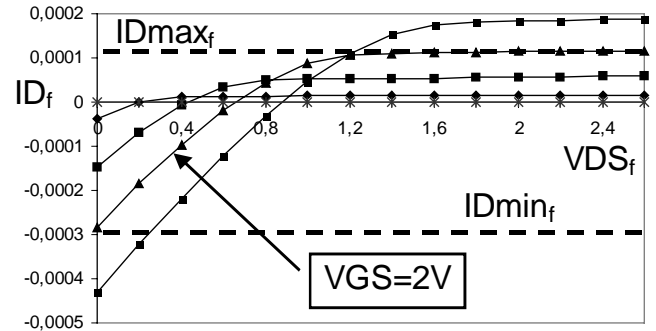

(a) ID vs VDS characteristics

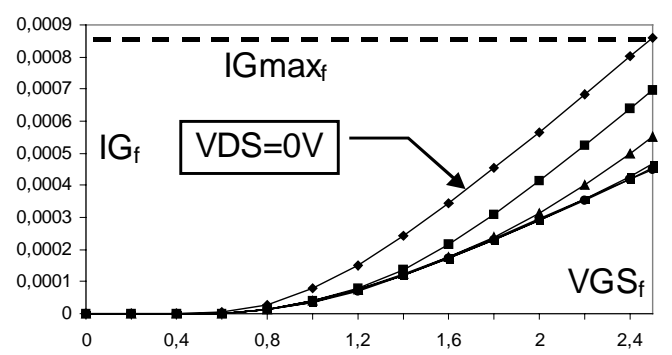

(b) IG vs VGS characteristics

Fig.8: Simulated I-V charateristics of a defective NMOS using the split model

\subsection{Limitations}

$A$ s described in the previous section, the split MOS model for GOS perfectly describes the behavior of gateto-channel defects and for this reason has been widely studied by different authors [8-14]. However this model suffers from severe limitations as discussed below. 
As everybody knows, SPICE simulations are performed using card models describing the transistor parameters in the targeted technological process. Using these card models, transistors of any length can be simulated as far as they are larger than the minimum $\left(\mathrm{L}_{\mathrm{min}}\right)$ allowed by the technological process. Any attempt to simulate transistors smaller than the minimum would make the simulation results (if any) unreliable.

Consequently, when using the split MOS model for simulating GOS defects, the elementary MOS transistor obtained after the splitting of the original transistor cannot have a length smaller than $\mathrm{L}_{\text {min }}$. This obviously implies that the original transistor cannot be at minimum length. Even the smallest network composed of two transistors in series as suggested in [13-14] corresponds to an original transistor with length equal or higher that $2 \mathrm{~L}_{\min }$. It is therefore impossible to study minimum transistors affected by GOS failures using a split MOS model. This is an extremely strong limitation taking into account that in a classical digital cell library, all transistors are designed at minimum length. In this context, although the split MOS transistor permits to accurately model the defect behavior, it clearly appears inadequate to simulate GOS defects in realistic digital circuits.

\section{Non-Linear Non-Split MOS Model}

This section describes the new model we developed for GOS defects presenting a non-linear behavior, i.e. gate-to-channel shorts. Our first objective is not to split the original transistor as proposed in [15] for the linear model in order to preserve the length of the original transistor. Our second objective is to introduce non-linearity in the model in order to properly represent the behavior of this type of defect. Consequently, our nonlinear model is constructed by adding non-linear components to the original transistor.

For the demonstration, we reuse the original transistor of figure 5 with $\mathrm{L}_{0}=2.1 \mu \mathrm{m}$ and $\mathrm{W}_{\mathrm{o}}=3.5 \mu \mathrm{m}$ in a $0.25 \mu \mathrm{m}$ technology. Because the split model has been proved very accurate [8-9], we consider that the characteristics of the faulty transistors are given by figure 8 . Note that these characteristics obtained with the split model are just used here as a reference.

Our non-linear non-split MOS model is now constructed in three steps with the aim to mimic as precisely as possible the characteristics of figure 8 .

\section{$\underline{\text { Step } 1}$}

As commented in section 2.2 , a first specificity of a transistor affected by a GOS failure resides in the reduction of the drain current. For a given VGS, we observe that the maximum drain current in the defective transistor is smaller than the maximum drain current in the non-defective one: $\operatorname{IDmax}_{\mathrm{f}}<\operatorname{IDmax}_{\mathrm{o}}$ (see figure 6 and 8). Consequently, we start our model construction with a transistor with the same length $\mathrm{L}_{\mathrm{o}}$ than the original transistor but a smaller width $\mathrm{W}_{\mathrm{m}}$ (W modified) to reduce the drain current. Note that the length of $\mathrm{L}_{\mathrm{o}}$ of the original transistor is preserved as required by our objectives; only the width $\mathrm{W}_{\mathrm{m}}$ is modified and calculated to match the faulty current $\operatorname{IDmax}_{\mathrm{f}}$. Figure 9 illustrates this first step.
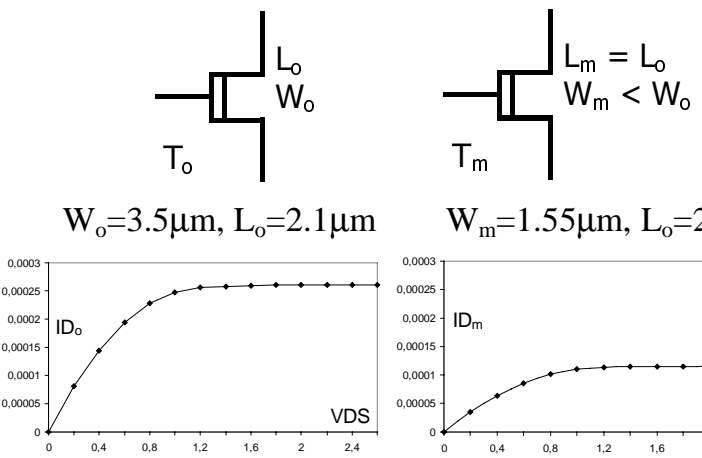

$\mathrm{W}_{\mathrm{m}}=1.55 \mu \mathrm{m}, \mathrm{L}_{\mathrm{o}}=2.1 \mu \mathrm{m}$

(a) original transistor

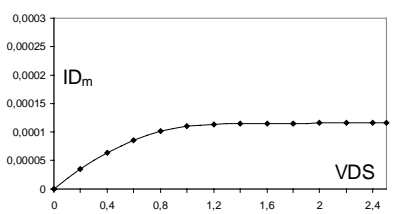

Fig.9: Non-linear non-split model - Step 1

\section{$\underline{\text { Step } 2}$}

The second specificity of a transistor affected by a GOS failure is the appearance of a negative drain current for low VDS. Consequently, the second step of our model construction consists in connecting an additional component allowing a current to flow from the gate to the drain. This additional component should act only at low VDS but not modify the I-V characteristics at high VDS. We propose to insert an additional transistor $T_{a}$ connected as illustrated in figure 10 .

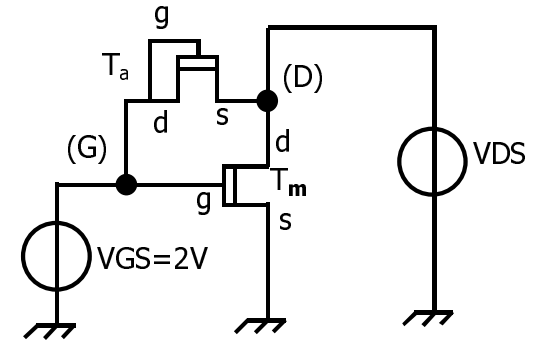

Fig.10: Non-linear non-split model - Step 2

For the sake of clarity, the behavior of the above circuit is analyzed for $\mathrm{VGS}=2 \mathrm{~V}$. The results will be then extended to any VGS.

When VDS is higher than $\mathrm{VGS}=2 \mathrm{~V}$, the additional transistor $T_{a}$ is OFF and has no influence on the I-V characteristics. When VD is smaller than $\mathrm{VGS}=2 \mathrm{~V}$, the additional transistor $T_{a}$ has its drain in node $G$ and its source in node D as illustrated in figure 10. Under these conditions, the transistor is $\mathrm{ON}$ and operates as a rectifier with $\operatorname{Vgd}_{\mathrm{a}}=0$. The corresponding $\mathrm{Ids}_{\mathrm{a}}$ vs $\mathrm{Vds}_{\mathrm{a}}$ characteristics are given in figure 11.a. It is to note that $\mathrm{Vds}_{\mathrm{a}}=\mathrm{VGS}-\mathrm{Vds}_{\mathrm{m}}$ so in figure 11.b and 11.c the 
characteristics are flipped and shifted to have compatible $\mathrm{x}$-axis with figure $8 . \mathrm{a}$.

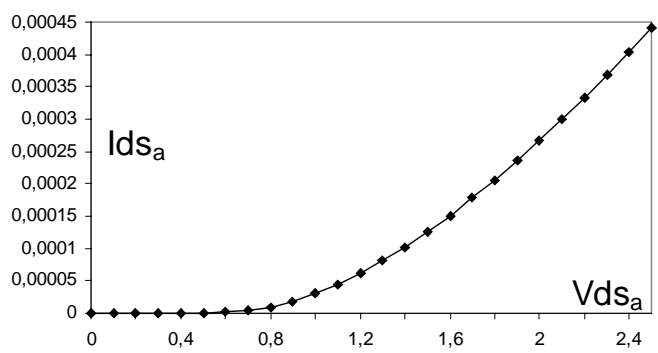

(a) $\mathrm{Ids}_{\mathrm{a}} \mathrm{Vs} \mathrm{Vds}_{\mathrm{a}}$

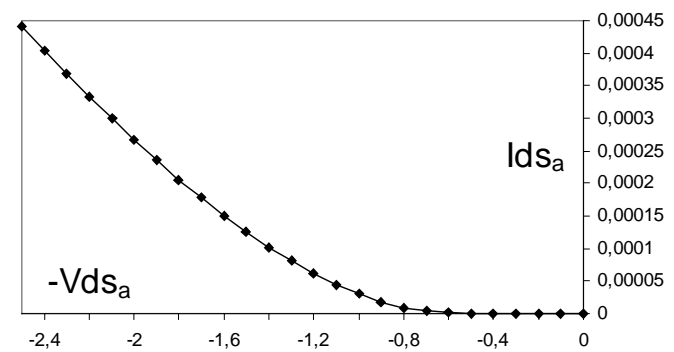

(b) $\operatorname{Ids}_{\mathrm{a}}$ vs $\left(-\mathrm{Vds}_{\mathrm{a}}\right)$

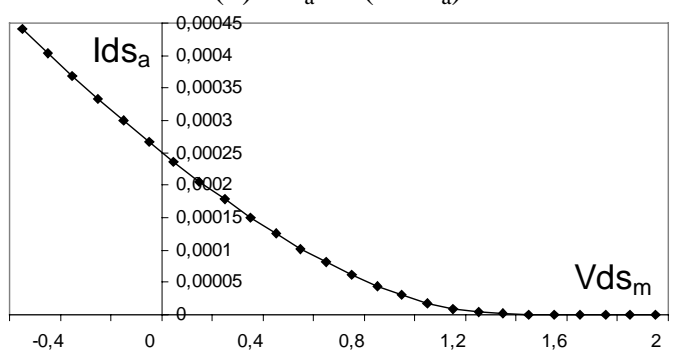

(c) $\mathrm{Ids}_{\mathrm{a}} \mathrm{Vs} \mathrm{Vds}_{\mathrm{m}}=\left(\mathrm{VGS}-\mathrm{Vds}_{\mathrm{a}}\right)$

Fig.11: I-V characteristics of the additional transistor $\mathrm{T}_{\mathrm{a}}$

In node $\mathrm{D}$, we can write the current equation as follows:

$$
\mathrm{ID}_{\mathrm{f}}+\mathrm{Ids}_{\mathrm{a}}=\mathrm{Ids}_{\mathrm{m}} \Rightarrow \mathrm{ID}_{\mathrm{f}}=\mathrm{Ids}_{\mathrm{m}}+\left(-\mathrm{Ids}_{\mathrm{a}}\right)
$$

That means that the current $\mathrm{ID}_{\mathrm{f}}$ flowing in the complete device can be obtained by adding the two characteristics of figure 12.a and 12.b. The resulting $\mathrm{ID}_{\mathrm{f}}$ current is represented in figure 12.c.

It is now just necessary to adjust the value of the IDmin $_{\mathrm{f}}$ current of figure 12.c to exactly fit the current IDmin $_{\mathrm{f}}$ of figure 8.a. The value of this current actually just depends on the additional transistor size $\mathrm{T}_{\mathrm{a}}$ since the current $\mathrm{Ids}_{\mathrm{m}}$ is equal to 0 at point VDS $=0$. Consequently, the current $\operatorname{IDmin}_{\mathrm{f}}$ in our model is simply set by tuning the $\mathrm{W}_{\mathrm{a}} / \mathrm{L}_{\mathrm{a}}$ parameter of the additional transistor $\mathrm{T}_{\mathrm{a}}$. Note that in figure 12.c, the $\mathrm{W}_{\mathrm{a}} / \mathrm{L}_{\mathrm{a}}$ parameter has been tuned to match the IDmin $_{\mathrm{f}}$ value of figure 8.a with $\mathrm{W}_{\mathrm{a}}=3.6 \mu \mathrm{m}$ and $\mathrm{L}_{\mathrm{a}}=2.1 \mu \mathrm{m}$.

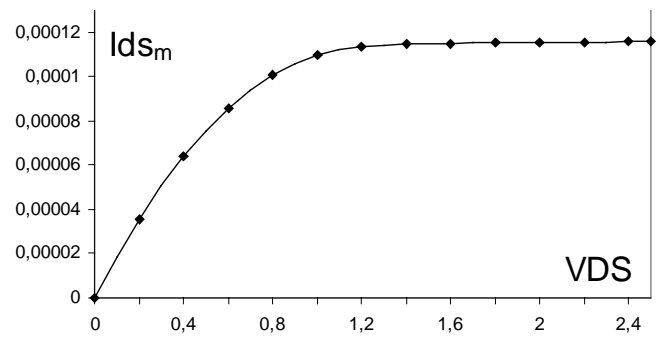

(a) $\operatorname{Ids}_{\mathrm{m}}$ vs VDS

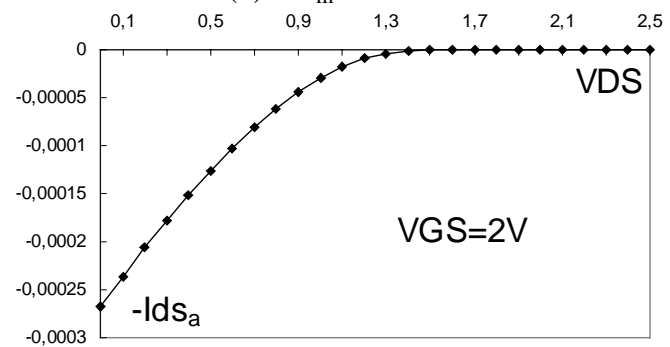

(b) $-\mathrm{Ids}_{\mathrm{a}}$ vs VDS

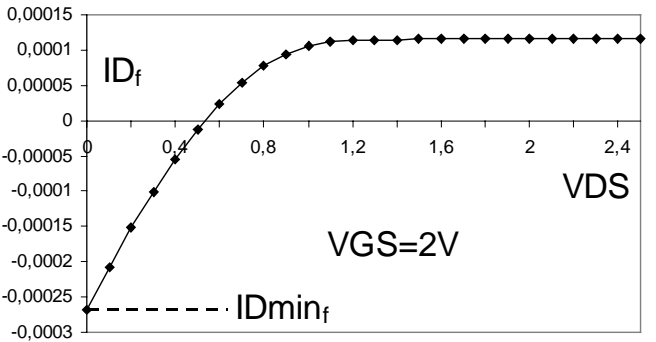

(c) $\mathrm{ID}_{\mathrm{f}} \mathrm{vs} \mathrm{VDS}$

Fig.12: I-V curves in the non-linear non-split model

The same demonstration can be made for any potential VG giving the complete set of $\mathrm{ID}_{\mathrm{f}}$ vs VDS characteristics of our non-linear non-split MOS model as presented in figure 13. It clearly appears that this model with its additional transistor correctly mimics the ID-VDS behavior of a gate-to-channel GOS.

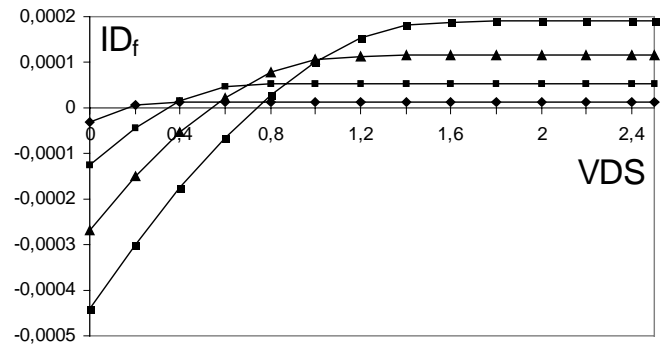

Fig.13: $\mathrm{ID}_{\mathrm{f}}$ vs VDS characteristics of the non-linear non-split model

\section{$\underline{\text { Step } 3}$}

Finally, the last specificity of a transistor affected by a gate-to-channel short is the presence of a non-linear gate current. Consequently, the last step in our model construction consists in connecting another additional transistor $\mathrm{T}_{\mathrm{b}}$ allowing a current to flow from the gate to the source, as illustrated in figure 14. 


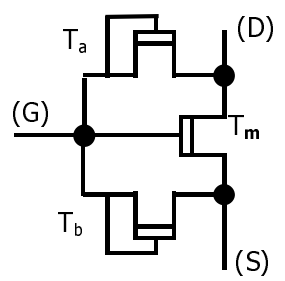

Fig.14: Non-linear non-split model - Step 3

This second additional transistor actually acts symmetrically to the first additional transistor, creating an IG current in the model. As performed in the previous step with the IDmin ${ }_{\mathrm{f}}$ current, we adjust the $\mathrm{W}_{\mathrm{b}} / \mathrm{L}_{\mathrm{b}}$ conductance of the second additional transistor $\mathrm{T}_{\mathrm{b}}$ to mimic the $\mathrm{IG}_{\mathrm{f}} \mathrm{vs}$ $\mathrm{VGS}_{\mathrm{f}}$ characteristics of figure 8.b. For example, when VDS $=0$, the current IG is maximum and figure 15 gives the equivalent circuit. The current $\mathrm{IGmax}_{\mathrm{f}}$ is equal to $\mathrm{Ids}_{\mathrm{a}}+\mathrm{Ids}_{\mathrm{b}}$. The current Ids $\mathrm{a}$ has already been fixed and so IGmax $_{f}$ is adjusted through $\mathrm{W}_{\mathrm{b}} / \mathrm{L}_{\mathrm{b}}$. To match the $\mathrm{IGmax}_{\mathrm{f}}$ value of figure 8.b, we set the additional transistor $T_{b}$ to with $\mathrm{W}_{\mathrm{b}}=3.6 \mu \mathrm{m}$ and $\mathrm{L}_{\mathrm{b}}=2.1 \mu \mathrm{m}$.

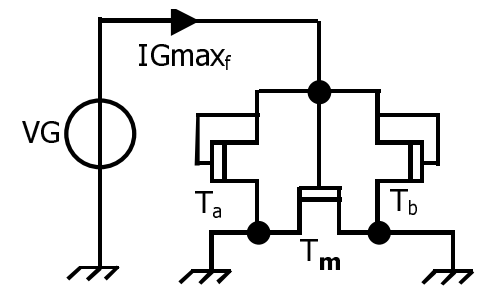

Fig.15: Equivalent circuit for the model at VDS $=0$

Finally, figure 16 gives the complete set of the IG vs VGS characteristics simulated with our model. These characteristics correctly reproduce the IG-VGS behavior of a gate-to-channel GOS.

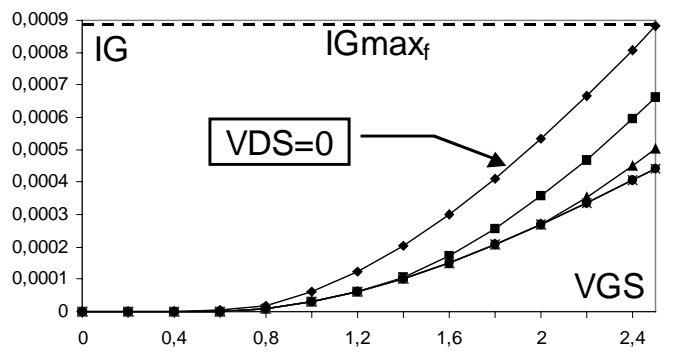

Fig.16: $\mathrm{IG}_{\mathrm{f}}$ vs VGS of the non-linear non-split model

\section{Conclusions}

$I_{\mathrm{n}}$

this paper, we proposed a new non-linear non-split MOS model for gate-to-channel GOS defects. The fundamental idea is not to split the original transistor in order to handle minimal-length transistors and therefore enable the simulation of GOS defects in realistic digital circuits. Based on the analysis of the electrical defect behavior, we developed a comprehensive method for the construction of the model. The model is constructed by first modifying the width of the original transistor, and then inserting two additional transistors between the gate and drain/source of this transistor:

- the width modification $\left(\mathrm{W}_{\mathrm{m}}\right)$ of the original transistor allows to adjust the level of the drain current at high VDS $\left(\right.$ IDmax $_{\mathrm{f}}$ ),

- the conductance $\mathrm{W}_{\mathrm{a}} / \mathrm{L}_{\mathrm{a}}$ of the first additional transistor allows to adjust the level of the negative drain current at low VDS $\left(\right.$ IDmin $\left._{\mathrm{f}}\right)$,

- the conductance $\mathrm{W}_{\mathrm{b}} / \mathrm{L}_{\mathrm{b}}$ of the second additional transistor allows to adjust the level of the gate current $\left(\mathrm{IG}_{\mathrm{f}}\right)$.

It has been shown that the behavior of the proposed model matches in a satisfactory way the behavior of a defective transistor model.

Finally, future work will concentrate on the tuning of the model parameters $\left(\mathrm{W}_{\mathrm{m}}, \mathrm{W}_{\mathrm{a}} / \mathrm{L}_{\mathrm{a}}, \mathrm{W}_{\mathrm{b}} / \mathrm{L}_{\mathrm{b}}\right)$ to reproduce GOS defects of various resistance, location and size.

\section{References}

[1] C.F. Hawkins, J.M. Soden, "Electrical Characteristics and Testing Considerations for Gate Oxide Shorts in CMOS ICs", Int. Test Conf., pp. 544-555, 1985.

[2] J.M. Soden, C.F. Hawkins, "Test considerations for Gate Oxide Shorts in CMOS ICs", Design \& Test of Computers, pp. 56-64, 1986.

[3] C.F. Hawkins, J.M. Soden, "Reliability and Electrical Properties of Gate Oxide Shorts in CMOS ICs", Int. Test Conf., pp. 443-451, 1986.

[4] S.I. Syed, D.M. Wu, "Defect Analysis, Test Generation and Fault Simulation for Gate Oxide Shorts in CMOS ICs", Int. Symp. Circuits and Syst., pp. 2705-2707, 1990.

[5] R. Rodriguez-Montanes, J. Segura, V. Champac, J. Figueras, J. Rubio, "Current vs. Logic Testing of Gate Oxide Short, Floating Gate and Bridging Failures", Int. Test Conf., pp. 510-519, 1991.

[6] J. Segura, J. Figueras, A. Rubio, "Approach to the Analysis of Gate Oxide Shorts in CMOS Digital Circuits", Microeletron. Reliab., Vol. 32, $\mathrm{N}^{\circ} 11$, pp. 1509-1514, 1992.

[7] J. Segura, C. De Benito, A. Rubio, C.F. Hawkins, "A Detailed Analysis of GOS Defects in MOS Transistors: Testing Implications at Circuit Level", Int. Test Conf., pp. 544-551, 1995.

[8] M. Renovell, J.M. Gallière, F. Azaïs, Y. Bertrand, "A Complete Analysis of the Voltage Behaviour of MOS Transistor with Gate Oxide Short", Defect-Based Testing Work., pp. 5-10, 2001.

[9] M. Renovell, J.M. Gallière, F. Azaïs, Y. Bertrand, "Analysing the Characteristics of MOS Transistors in the Presence of Gate Oxide Short", Design \& Diag. of Electr. Circuits and Syst., pp. 155-161, 2001 [10]M. Renovell, J.M. Gallière, F. Azaïs, Y. Bertrand, "Boolean and Current Detection of MOS Transistor with Gate Oxide Short", Int. Test Conf., pp. 1039-1048, 2001

[11]M. Syrzycki, "Modeling of Spot Defects in MOS Transistors", Int. Test Conf., pp. 148-157, 1987.

[12]M. Syrzycki, "Modeling of Gate Oxide Shorts in MOS Transistors", Trans. on Computer-Aided Design, Vol. 8, pp. 193-202, 1989.

[13]J. Segura, A. Rubio, J. Figueras, "Analysis and Modeling of MOS Devices with Gate Oxide Short Failures", Int. Symp. Circuits and Syst., pp. 2164-2167, 1991.

[14]V. Champac, R. Rodriguez-Montanes, J. Segura, J. Figueras, J. Rubio, "Fault Modeling of Gate Oxide Short, Floating Gate \& Bridging Failures in CMOS Circuits", Europ. Test Conf., pp. 143-148, 1991.

[15]H. Hao, E.J. McCluskey, "On the Modeling and Testing of Gate Oxide Shorts in CMOS Logic Gates", Int. Work. on Defect and Fault Tolerance on VLSI Systems, pp. 161-174, 1991.

[16]H. Hao, E.J. McCluskey, "Analysis of Gate Oxide Shorts in CMOS Circuits", Trans. on Computers, Vol. 42, pp. 1510-1516, 1993.

[17]J. Segura, C. De Benito, A. Rubio, C.F. Hawkins, "A Detailed Analysis and Electrical Modeling of Gate Oxide Shorts in MOS Transistors", JETTA, Nº , pp. 229-239, 1996. 